\title{
The Design of Intelligent Speech Walking Stick Based on GPS
}

\author{
Yuge Wang and Ling Long \\ Southwest Minzu University, College of Electrical and Information Engineering, 610041, Chengdu, China
}

\begin{abstract}
With the development of society and the progress of science and technology, the traditional walking stick has been unable to meet the needs of modern walking stick users. Based on this, we developed the intelligent speech navigation walking stick design based on GPS and GSM, supplemented by the functions of ultrasonic obstacle avoidance, night warning and emergency assistance. This walking stick can help the blind walk on the road independently. Moreover, when the user cannot distinguish the road information, the walking stick can realize the real-time voice navigation function in the region, so as to solve the problem that the user cannot independently find the road information.
\end{abstract}

\section{Introduction}

With the development of modern society, there are various kinds of guide canes in the market. After careful investigation, it can be found that these products are less helpful for blind people to travel outside freely[1]. Therefore, we have developed a design of intelligent voice navigation walking stick based on GPS and GSM, which can guide blind people to their designated destinations accurately and effectively improve their travel problems.

\section{Overall system design}

The walking stick is mainly controlled by the microcontroller STM32F4, supplemented by four modules: ultrasonic obstacle avoidance, voice navigation, night warning and emergency short message transmission. The structure diagram is shown in figure 1.

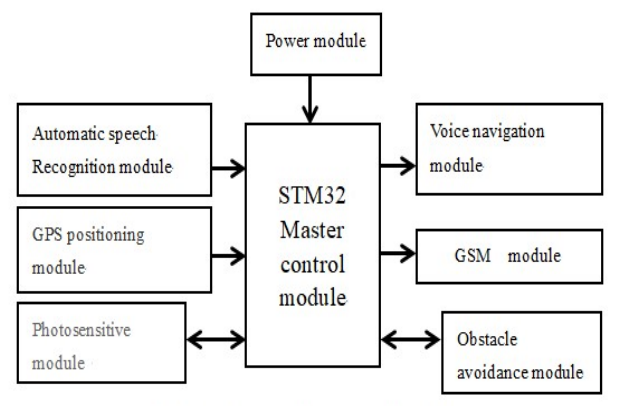

Figure 1. The overall system block diagram
When the user speaks the specified destination, the cane will be informed of its current location according to the GPS positioning module, and its walking route will be obtained through the algorithm and voice broadcast. At the same time, the light-sensitive module on the walking stick will automatically turn on the warning light in the dark to prevent other pedestrians from hitting the user. The obstacle avoidance module facilitates users to avoid obstacles and walk safely. When the user encounters an emergency, he or she can press the button and use the GSM module to send a text message, positioning himself or herself to the designated mobile phone number for a call.

\section{The hardware design}

\subsection{GPS positioning \&GSM module}

The A7 GSM/GPRS/GPS module can support GPS positioning, voice and SMS transmission. The U_TXD, GPS_TXD and GND pins of the module are connected to PA2, PA3, and GND pins of the master chip respectively, and the designated user can be dialed with the SIM card, thus realizing the emergency call function of the walking stick. After connecting the U_RXD pin of the module to the RX1 pin of the master chip, the position information collected by the module can be returned to the master chip through the serial port. After the master chip reads its information, the position information can be further processed, and finally the circuit design of GPS positioning and voice navigation functions can be realized $[2,3]$. 


\subsection{Voice module}

The module uses the LD3320 module, which is a nonspecific speech recognition chip produced by ICRoute company. The so-called non-specific speech refers to that the speech recognition is not specific to the tone of a particular tone, it does not need to carry on the phonetic training, even if people of different ages and genders, as long as they speak the same phonetic word, it can be recognized, recognition accuracy as high as 95\%.Its function is to transmit the signal to the MCU after speech recognition, and get the destination it wants to reach through the conversion of the main control chip, so as to design the route[4]. The Speech navigation module block diagram is shown in figure 2 .

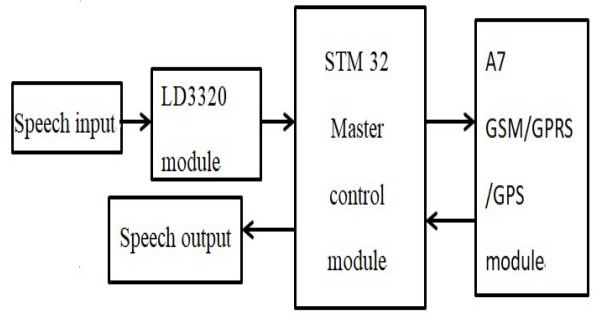

Figure 2. The Speech navigation module block diagram

\subsection{Ultrasonic obstacle avoidance module}

This module adopts hc-sr04 module, whose function is to measure the distance between users and obstacles through ultrasonic wave [5].When the distance is less than $150 \mathrm{~cm}$, the module will send a signal, connect with the master chip through the serial port, send the signal to the master chip, trigger the buzzer, and indicate the obstacle in front of the user through the sound, so as to achieve the function of obstacle avoidance [6].

\subsection{Photosensitive module}

This module uses a photoresistance module, which can realize the function of recognizing the light and dark of the whole environment[7]. The DO output terminal is directly connected to the master chip to detect the high and low level through the master chip, so as to detect the changes in the light intensity of the environment. When the module detects a dim environment, the cane automatically lights up a warning light to alert passers-by and prevent cars or people from hitting the user.

\section{The software design}

The program part of the system is written by $\mathrm{C}$ language modules, including ultrasonic obstacle avoidance, speech recognition, speech broadcast and GPS positioning module[8].The stick is designed with three buttons: the master switch, the emergency call key $\mathrm{A}$ and the navigation key B. this makes the design leaner and avoids making the program more cumbersome. The system software design block diagram is shown in figure 3. As a result, the system is divided into three modes, as follows:

\subsection{Mode 1}

The system starts when only the master switch button is pressed, but it only has the function of obstacle avoidance and night warning. All modules of the system are initialized, and the ultrasonic obstacle avoidance module and the photosensitive module begin to work at the same time. Start timer T0 and Send the ultrasonic wave, and when the return wave is received, turn off the timer and calculate the obstacle with the duration of high level Distance. When the distance is less than $150 \mathrm{~cm}$, the buzzer is triggered. And when the D0 end of the photosensitive module output high level Turn on the warning light.

\subsection{Mode 2}

While pressing the emergency call button $A$, the function of dialing the phone can be used. When the state of key A is changed, the interrupt can be triggered, and the main control chip sends "ATD+ number +;"The SIM card can be used to call the phone for help.

\subsection{Mode 3}

When you press the navigation button B, you can use the voice navigation function. When the state of button B is changed, it will be interrupted. The main control chip sends "AT+GPS=1" to enable the GPS function. Then input "\$GPGGA" command to obtain the GPS positioning information $[9,10]$. 


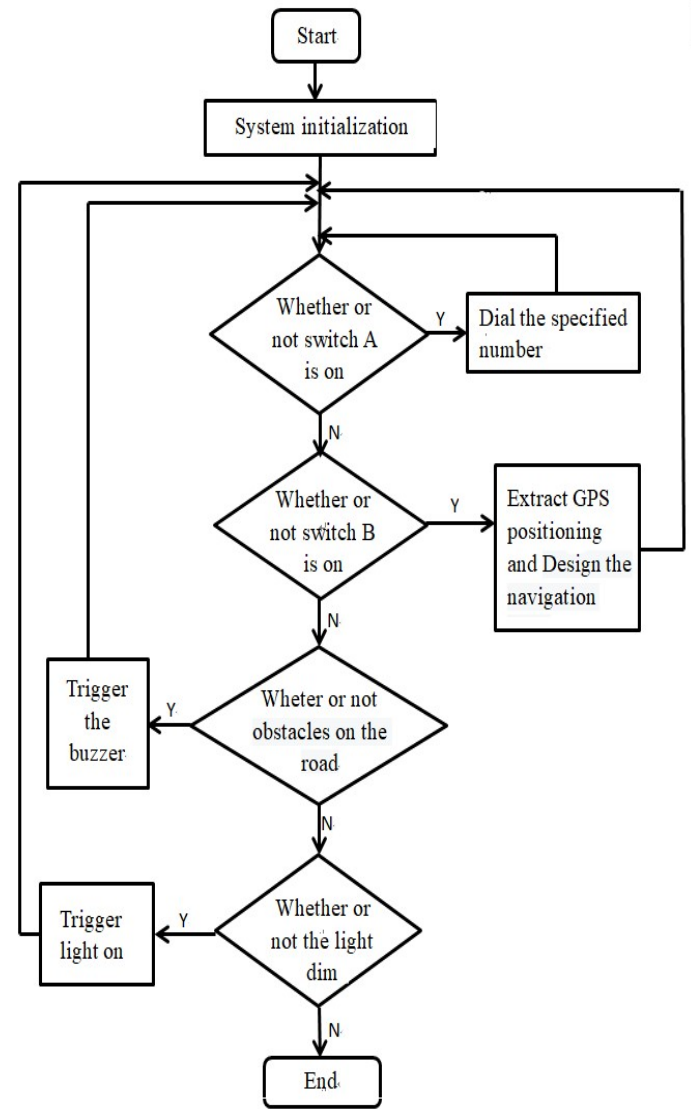

Figure 3. The system program flow chart

\section{Summary}

The system is an intelligent navigation walking stick based on STM32, which uses GPS positioning technology to collect geographical position. The idea of intelligent navigation in the region is realized through the algorithm in the system, supplemented by various modules. Finally, a smart walking stick that integrates voice navigation, obstacle avoidance, night warning and emergency call is realized. The walking stick has the advantages of humanization, intelligence and simple operation. It can effectively help the elderly and the blind to travel daily and improve the quality of their living.

\section{Acknowledgements}

This work was financially supported by the Fundamental Research Funds for the Central Universities of Southwest Minzu University (NO. 201810656013).

\section{References}

1. Hui-fu Zhang; Wei Kang, Design of the Data Acquisition System Based on STM32, Procedia Computer Science, 5 (2013)

2. ELLIONTT D, KAPLAN, GPS Principle and Application(2002)

3. Hui-nan WANG, GPS Navigation Principle and Application, (2003)

4. Kan He, Ya-qing Tian, Qiang Li, Zhou-rong Hu, Jing Zhang, Design of speech-recognition intelligent trash based on LD3320, Foreign Electronic Measurement Technology,6(2015)

5. Takanori EMARU, Takeshi, TSUCHIYA, Research on estimating the smoothed value and different value of the distance measured by an ultrasonic wave sensor, Proceedings of the IEEE International Conference on Intelligent Robets and System (2000)

6. Li Xu, Sheng-nan Cao, Li-wei Tang, The Design and Realization of the Intelligent Walking Stick Based on AT89C51 Chip Microcomputer, Journal of Taiyuan Normal University(Natural Science Edition),4 (2009)

7. Dong Yang, Ke-hui Xuan, Xue-feng Dong, Characteristics and Applications of Photosensitive Resistance, Journal of Shandong Polytechnic University(Natural Science Edition), 2(2013)

8. Brian Nagel, Micrium, Advantages of the CortexM3, Information Quarterly,(2008)

9. Jia Zhao, Jun-yu Yang, Ya-qiu Li, Vehicle Positioning Monitor and Control System Based on GSM and GPS, Meteorological,Hydrological and Marine Instruments, 4(2007)

10. Misra, The Role of the Clock in the GPS Receiver, GPS World(1996) 